\title{
In vitro and in vivo anti-primary effusion lymphoma activities of fucoidan extracted from Cladosiphon okamuranus Tokida
}

\author{
CHIE ISHIKAWA $^{1,2}$ and NAOKI MORI ${ }^{1}$
}

\author{
${ }^{1}$ Department of Microbiology and Oncology, Graduate School of Medicine, University of the Ryukyus, \\ Nishihara, Okinawa 903-0215; ${ }^{2}$ Division of Health Sciences, Transdisciplinary Research Organization \\ for Subtropics and Island Studies, University of the Ryukyus, Nishihara, Okinawa 903-0213, Japan
}

Received May 19, 2017; Accepted September 11, 2017

DOI: $10.3892 /$ or.2017.5978

\begin{abstract}
Primary effusion lymphoma (PEL) caused by Kaposi's sarcoma-associated herpesvirus (KSHV) is characterized by lymphomatous effusion in body cavities and poor prognosis. There is still no effective treatment for PEL. Fucoidan, a major sulfated polysaccharide isolated from brown seaweeds, has an attractive array of bioactivities such as cancer inhibition. However, the effects of fucoidan on PEL cells remain unclear. We investigated the anti-PEL effects of fucoidan obtained from Cladosiphon okamuranus Tokida cultivated in Okinawa. Fucoidan dose-dependently inhibited the proliferation of KSHV-infected PEL cell lines, and provoked $\mathrm{G}_{1}$ cell cycle arrest, which was accompanied by CDK4 and CDK6 downregulation. Fucoidan also induced apoptosis of PEL cells through caspase-3, -8 and -9 activation; this occurred partly through the downregulation of anti-apoptotic Bcl-xL, Mcl-1 and XIAP proteins. Fucoidan also suppressed nuclear factor- $\kappa \mathrm{B}$, activator protein-1 (AP-1), and T-lymphokineactivated killer cell-originated protein kinase (TOPK) signaling pathways through inhibition of phosphorylation of $I \kappa \mathrm{B} \alpha$ and TOPK, and the expression of AP-1 family proteins, JunB and JunD. Oral administration of fucoidan effectively inhibited the development of PEL cells and ascites in a xenograft SCID mouse model, with minimal serious adverse effects. Notably, native fucoidan exhibited a more efficient anti-PEL effect than nanoparticle fucoidan. These preclinical findings highlight the anti-PEL actions of fucoidan, suggesting it could be potentially useful for the prevention and treatment of PEL.
\end{abstract}

\section{Introduction}

Primary effusion lymphoma (PEL, also termed body cavity lymphoma) is a highly aggressive non-Hodgkin's B cell

Correspondence to: Professor Naoki Mori, Department of Microbiology and Oncology, Graduate School of Medicine, University of the Ryukyus, 207 Uehara, Nishihara, Okinawa 903-0215, Japan E-mail: naokimori50@gmail.com

Key words: fucoidan, primary effusion lymphoma, nuclear factor- $\kappa \mathrm{B}$, activator protein-1, T-lymphokine-activated killer celloriginated protein kinase lymphoma associated with poor prognosis that arises frequently in immune-compromised individuals, such as organ transplantation recipients and the HIV-infected population $(1,2)$. PEL is strictly associated with Kaposi's sarcoma-associated herpesvirus (KSHV) and characterized by lymphomatous effusions of pleural, pericardial and abdominal cavities in the absence of tumor masses $(1,2)$. It is generally resistant to conventional chemotherapy with a short median survival of less than 6 months. Furthermore, the myelosuppressive effects of systemic cytotoxic chemotherapy synergize with those caused by antiretroviral therapy or immune suppression $(3,4)$. Therefore, new therapeutic strategies are needed for PEL.

PEL cell survival relays on the constitutive activation of several pathways. These include nuclear factor $-\kappa \mathrm{B}(\mathrm{NF}-\kappa \mathrm{B})$ and activator protein-1 (AP-1) (5-8). The KSHV-encoded viral FLICE-inhibitory protein and $\mathrm{G}$ protein-coupled receptor mediate activation of $\mathrm{NF}-\kappa \mathrm{B}$ and AP-1 pathways (6-8). It has been reported that inhibition of $\mathrm{NF}-\kappa \mathrm{B}$ significantly decreases PEL cell survival (5).

Fucoidan, a sulfated polysaccharide, is abundant in brown seaweeds. It is composed of L-fucose as well as other sugars, such as D-xylose, D-galactose, D-mannose and glucuronic acid (9). Several studies have reported that fucoidan possesses many desired biological effects, such as anticancer and antiviral activities $(9,10)$. Moreover, it is a well-tolerated agent $(11,12)$.

The present study was designed to determine the anti-PEL activity of fucoidan both in vitro and in vivo. The results revealed that fucoidan inhibited constitutively active $\mathrm{NF}-\kappa \mathrm{B}$, AP-1 and lymphokine-activated killer T-cell-originated protein kinase (TOPK), leading to $\mathrm{G}_{1}$ cell cycle arrest and apoptosis of PEL cells. Oral administration of fucoidan suppressed PEL progression in a xenograft murine model.

\section{Materials and methods}

Reagents. Fucoidan was prepared from the brown algae Cladosiphon okamuranus Tokida cultivated in Okinawa as previously described in detail (13). Nanoparticle fucoidan was also previously described (14). Fucoidan was dissolved in RPMI-1640 medium (cat. no. 30264-56, Nacalai Tesque, Inc., Kyoto, Japan). The primary antibodies against cleaved caspase-3 (cat. no. 9664), caspase-8 (cat. no. 9446), caspase-9 (cat. no. 9501) and poly(ADP-ribose) polymerase (PARP) 
(cat. no. 9541), Bcl-xL (cat. no. 2762), phospho-IкB $\alpha$ (Ser32 and 36) (cat. no. 9246), RelA (cat. no. 8242), TOPK (cat. no. 4942) and phospho-TOPK (Thr9) (cat. no. 4941) were obtained from Cell Signaling Technology, Inc. (Beverly, MA, USA). Antibodies against CDK4 (cat. no. MS-299), CDK6 (cat. no. MS-398), cyclin E (cat. no. MS-870), Bcl-2 (cat. no. MS-597) and actin (cat. no. MS-1295) were purchased from Neomarkers, Inc. (Fremont, CA, USA). An antibody against XIAP (cat. no. M044-3) was purchased from Medical \& Biological Laboratories, Co. (Aichi, Japan). Antibodies against cyclin D2 (cat. no. sc-593), Mcl-1 (cat. no. sc-819), c-IAP2 (cat. no. sc-7944), I $\mathrm{B} \alpha$ (cat. no. sc-371), lamin B (cat. no. sc-6216), JunB (cat. no. sc-46) and JunD (cat. no. sc-74), and NF- $\mathrm{BB}$ subunits p50 (cat. no. sc-114X), p52 (cat. no. sc-298X), RelA (cat. no. sc-109X), c-Rel (cat. no. sc-70X) and RelB (cat. no. sc-226X), and AP-1 subunits c-Fos (cat. no. sc-52X), FosB (cat. no. sc-48X), Fra-1 (cat. no. sc-605X), Fra-2 (cat. no. sc-604X), c-Jun (cat. no. sc-45X), JunB (cat. no. sc-46X) and JunD (cat. no. sc-74X) for supershift assays were obtained from Santa Cruz Biotechnology, Inc. (Santa Cruz, CA, USA).

Cells. The human B-cell lines derived from PEL, carrying KSHV, BCBL-1 (15) and TY-1 (16), were maintained in RPMI-1640 medium supplemented with $10 \%$ heat-inactivated fetal bovine serum (Biological Industries, Kibbutz Beit Haemek, Israel) and $1 \%$ penicillin/streptomycin (cat. no. 09367-34, Nacalai Tesque, Inc.) in a 5\% $\mathrm{CO}_{2}$ humidified incubator at $37^{\circ} \mathrm{C}$.

Cell proliferation and cytotoxic assay. PEL cells were seeded into 96-wells plates in triplicate at a density of $10^{4}$ cells/well and treated with various concentrations of fucoidan for $72 \mathrm{~h}$. The effects of fucoidan on cell proliferation and cytotoxicity were determined by the water-soluble tetrazolium (WST)- 8 uptake method. After incubation, $10 \mu \mathrm{l}$ of the WST- 8 reagent (cat. no. 07553-44, Nacalai Tesque, Inc.) were added to each well. After $4 \mathrm{~h}$, WST-8 reduction was measured at $450 \mathrm{~nm}$ using a 680 XR microplate reader (Bio-Rad Laboratories, Inc., Hercules, CA, USA). The percentage of cells was calculated by normalizing the optical density values of fucoidan-treated samples vs. the untreated control samples.

Apoptosis assay. PEL cells were treated with various doses of fucoidan for 24-72 $\mathrm{h}$ and then permeabilized by incubation on ice for $20 \mathrm{~min}$ with $100 \mu \mathrm{g} / \mathrm{ml}$ of digitonin, and treated with the phycoerythrin-conjugated APO2.7 antibody (cat. no. IM2088, Beckman Coulter, Inc., Marseille, France) for $15 \mathrm{~min}$ at room temperature. After staining with the APO2.7 antibody, apoptosis was determined using an Epics XL flow cytometer (Beckman Coulter, Inc., Brea, CA, USA).

In vitro assessment of caspase activity. Caspase activity was assessed using Colorimetric Caspase Assay kits (cat. nos. 4800, 4805 and 4810; Medical \& Biological Laboratories, Co.). Briefly, cell extracts were recovered using the cell lysis buffer supplied with the kits and assessed for caspase-3, -8 and -9 activities using colorimetric probes. The assay kits are based on the detection of chromophore Q-nitroanilide after cleavage from caspase-specific labeled substrates. Colorimetric readings were performed in an automated microplate reader.

Cell cycle analysis. Cells were stained with the CycleTEST Plus DNA Reagent kit (cat. no. 340242; Becton-Dickinson Immunocytometry Systems, San Jose, CA, USA). The cell cycle distribution was analyzed for 10,000 collected cells by an Epics XL flow cytometer equipped with MultiCycle software (version 3.0; Phoenix Flow Systems, San Diego, CA, USA). The population of nuclei in each phase of the cell cycle was determined.

Western blot analysis. Whole cell extracts were prepared by subjecting fucoidan-treated cells to lysis in lysis buffer [62.5 mM Tris-HCl (pH 6.8) (cat. no. 35434-21), 2\% sodium dodecyl sulfate (cat. no. 31607-65), 10\% glycerol (cat. no. 1704565), 6\% 2-mercaptoethanol (cat. no. 21438-82; all from Nacalai Tesque, Inc.) and $0.01 \%$ bromophenol blue (cat. no. 021-02911; Wako Pure Chemical Industries, Osaka, Japan)]. Lysates were spun to remove insoluble material. Supernatants were collected and protein concentrations were assessed. Protein lysates $(20 \mu \mathrm{g})$ were resolved by sodium dodecyl sulfatepolyacrylamide gel electrophoresis (SDS-PAGE). Then, the proteins were transferred onto polyvinylidene difluoride membranes (cat. no. IPVH00010EMD; Millipore, Darmstadt, Germany) and immunoblotted with relevant specific antibodies. Immunoreactive bands were identified by an enhanced chemiluminescence reagent (cat. no. RPN2232; Amersham Biosciences Corp., Piscataway, NJ, USA).

Electrophoretic mobility shift assay (EMSA). To determine $\mathrm{NF}-\kappa \mathrm{B}$ and AP-1 activation, we prepared nuclear extracts from fucoidan-treated cells and performed EMSA as previously described (17). The obtained nuclear extracts were also subjected to SDS-PAGE. The top strand sequences of the oligonucleotide probes or competitors were as follows: for a consensus NF- $\mathrm{KB}$ element of the interleukin-2 receptor $\alpha$ chain $(I L-2 R \alpha)$ gene, 5'-GATCCGGCAGGGGAATCTCCCT CTC-3' and for the typical AP-1 element of the $I L-8$ gene, 5'-GATCGTGATGACTCAGGTT-3'. The above underlined sequences are the $\mathrm{NF}-\kappa \mathrm{B}$ and $\mathrm{AP}-1$ binding sites, respectively. To determine the specificity of the binding, nuclear extracts were preincubated with 100 -fold excess of unlabeled oligonucleotides for $15 \mathrm{~min}$. For supershift assays, different antibodies against $\mathrm{NF}-\kappa \mathrm{B}$ or AP-1 subunits were preincubated with nuclear protein for $45 \mathrm{~min}$ at room temperature before the addition of probes. The dried gels were visualized.

PEL xenograft model. Aliquots of $5 \times 10^{6}$ TY-1 cells or $8 \times 10^{7}$ BCBL-1 cells were suspended in sterile RPMI-1640 medium (200 $\mu \mathrm{l})$, and 5-week-old female C.B-17/Icr-severe combined immune deficiency (SCID) mice (Kyudo, Co., Tosu, Japan) received intraperitoneal injections with a single-cell aliquot. Fucoidan (200 mg/kg for TY-1 and $150 \mathrm{mg} / \mathrm{kg}$ for BCBL-1), or vehicle alone, was administered using oral gavage once daily for 5 days per week, and the treatment was continued for 50 days (TY-1) and 56 days (BCBL-1), beginning the day of injection (TY-1) or one day after injection (BCBL-1). PEL expansion in vivo was confirmed by testing the expression of cell surface markers, including cluster of differentiation 30 
A

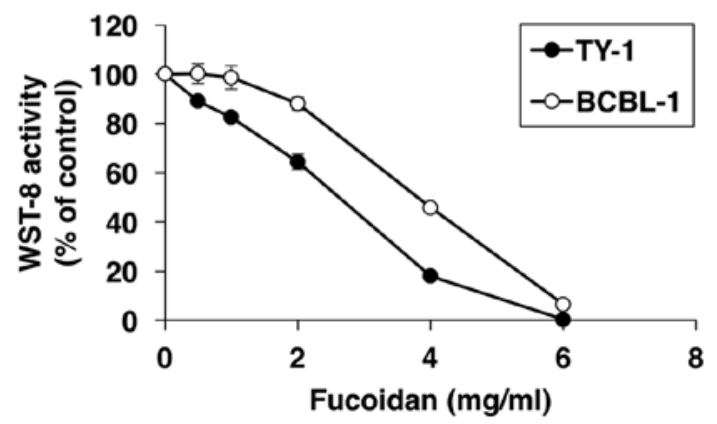

B

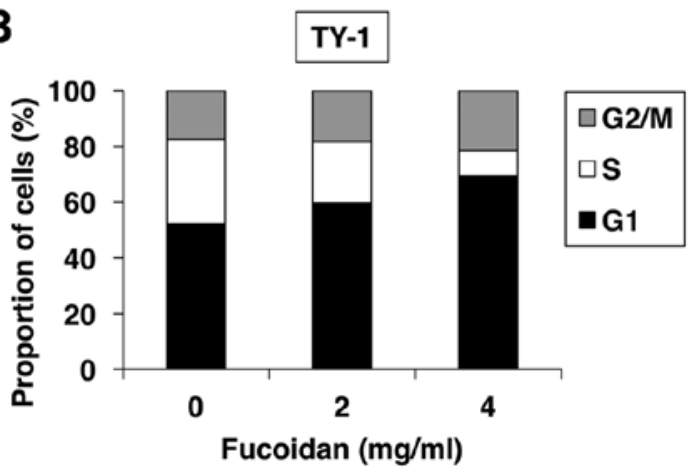

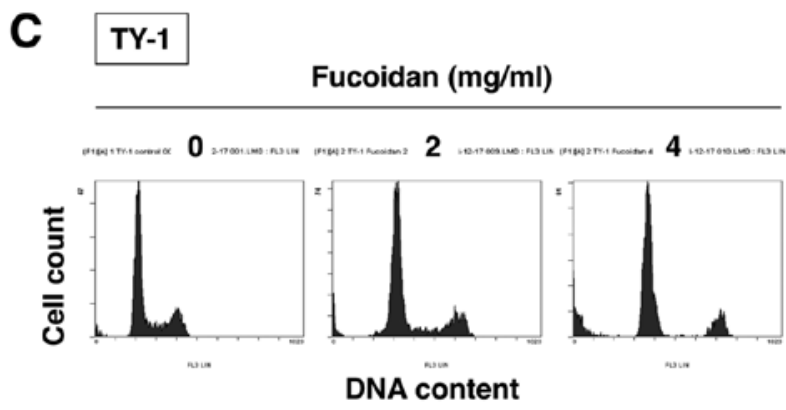

Figure 1. Fucoidan inhibits primary effusion lymphoma (PEL) cell proliferation. (A) TY-1 and BCBL-1 cells were treated with increasing concentrations of fucoidan for $72 \mathrm{~h}$ and then cell proliferation and cytotoxicity were assessed by WST-8 assay. Data are presented as the mean \pm SD ( $\mathrm{n}=3$ ). (B and C) Fucoidan inhibited PEL cell cycle progression. To determine the effect of fucoidan on cell cycle progression, TY- 1 cells were treated with fucoidan ( 2 and 4 mg/ml) for $24 \mathrm{~h}$, and analyzed for cell cycle distribution by flow cytometry. (B) Quantification of the percentage of cells at each phase of the cell cycle $\left(\mathrm{G}_{2} / \mathrm{M}, \mathrm{S}\right.$ and $\left.\mathrm{G}_{1}\right)$. (C) Representative data of the control and the treated cells from a single experiment conducted in triplicate with similar results.

(CD30) in ascites tumor cells, using flow cytometry. Body weight was recorded weekly as a surrogate measure of tumor progression. All experiments were performed in compliance with the Guidelines for Animal Experimentation of the University of the Ryukyus (Nishihara, Japan) and approved by the Animal Care and Use Committee of the University of the Ryukyus (reference nos. 5885 and A2016097).

Biomarker analysis. Serum and ascitic concentrations of human soluble CD30 (sCD30) (cat. no. RAF091R; BioVendor Inc., Brno, Czech Republic) were assessed by enzyme-linked immunosorbent assay (ELISA), according to the protocol supplied by the manufacturer.

Statistical analysis. Data are expressed as the mean \pm standard deviation (SD), unless otherwise stated. Data of two groups were compared with the Student's t-test. Differences were considered significant at the $95 \%$ confidence level when $\mathrm{P}<0.05$.

\section{Results}

Effects of fucoidan on PEL cell proliferation and apoptosis. The two different PEL cell lines, BCBL-1 and TY-1, were treated with increasing doses of fucoidan for $72 \mathrm{~h}$. Fucoidan decreased cell proliferation and survival in a dose-dependent fashion in both PEL cell lines, as assessed by WST-8 assay (Fig. 1A).

We also evaluated the effect of fucoidan on cell cycle regulation using flow cytometry. As shown in Fig. 1B and C, upon 24-h culture of fucoidan-treated TY-1 cells, the percentage of cells in the $G_{1}$ phase increased from $52.1 \%$ (in vehicle-treated cells) to $59.6 \%$ (in $2 \mathrm{mg} / \mathrm{ml}$ fucoidan-treated cells) and $69.3 \%$ (in $4 \mathrm{mg} / \mathrm{ml}$ fucoidan-treated cells), whereas the percentage of cells in the $\mathrm{S}$ phase decreased from $30.5 \%$ (in vehicle-treated cells) to $22.1 \%$ (in $2 \mathrm{mg} / \mathrm{ml}$ fucoidan-treated cells) and $7.5 \%$ (in $4 \mathrm{mg} / \mathrm{ml}$ fucoidan-treated cells). Thus, fucoidan enhanced accumulation in the $G_{1}$ phase of the cell cycle in a dose-dependent manner.

In the next step, we investigated the type of cell death induced by fucoidan and found that it increased the number of APO2.7-positive cells in dose- and time-dependent manners (Fig. 2A). APO2.7 antibody reacted with a 38-kDa mitochondrial membrane protein (named 7A6 antigen), which is detected in late apoptotic cells (18). These results revealed that fucoidan induced apoptotic cell death. The apoptotic process was executed by a member of the highly conserved caspase family (19). Western blot analysis revealed that fucoidan dosedependently induced the cleavage of initiators caspase- 8 and -9 , and the executioner caspase-3 and its substrate PARP (Fig. 2B). To confirm caspase activation by fucoidan for induction of apoptosis, ELISA was performed with various caspase substrates. PEL cells treated with fucoidan exhibited enhanced activation of caspase- 8 and -9 ; thereby activating effector caspase-3 (Fig. 2C).

Fucoidan induces downregulation of CDK4,CDK6, Bcl-xL, $M c l-1$ and XIAP. We further investigated the levels of various molecules involved in cellular proliferation and survival. Western blot analysis revealed dose-dependent downregulation of cyclin-dependent kinases (CDK4 and CDK6) and anti-apoptotic proteins Bcl-xL, Mcl-1 and XIAP in TY-1 cells treated with fucoidan (Fig. 3). 
A

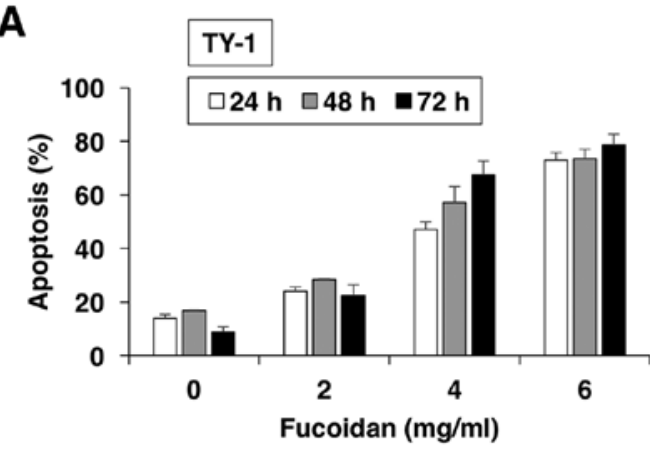

B

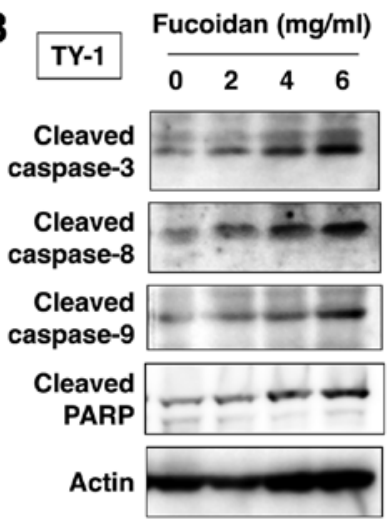

C

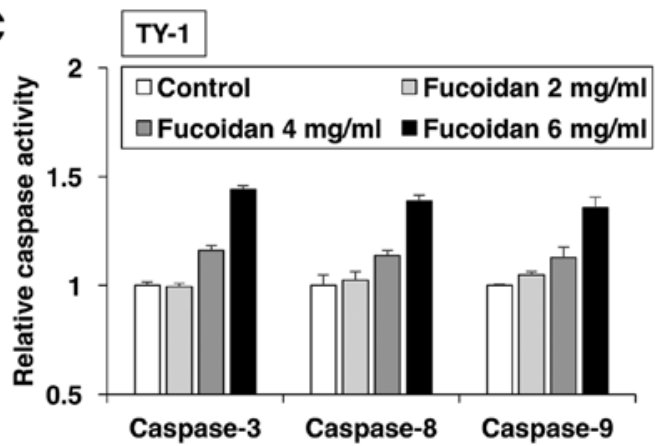

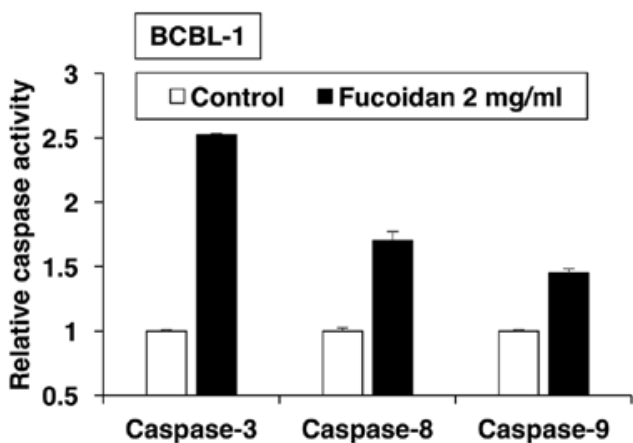

Figure 2. Fucoidan induces caspase-mediated cell apoptosis. (A) TY-1 cells were treated with different concentrations of fucoidan for 24-72 h, and flow cytometry was used to detect cell apoptosis by APO2.7 staining. Values are presented as the mean $\pm \mathrm{SD}(\mathrm{n}=3)$. (B) Cleaved caspase-3, $-8,-9$ and PARP levels were assessed by western blot analysis after treatment with different concentrations of fucoidan for $72 \mathrm{~h}$ and preparation of whole cell lysates. Actin was the internal control. (C) Caspase activity was assessed using relevant caspase colorimetric assay kits following treatment of TY-1 and BCBL-1 with the indicated concentrations of fucoidan for $72 \mathrm{~h}$. Data represent the relative caspase activity, which was expressed as multiples of induction beyond each respective control. Values are presented as the mean $\pm \mathrm{SD}(\mathrm{n}=3)$.
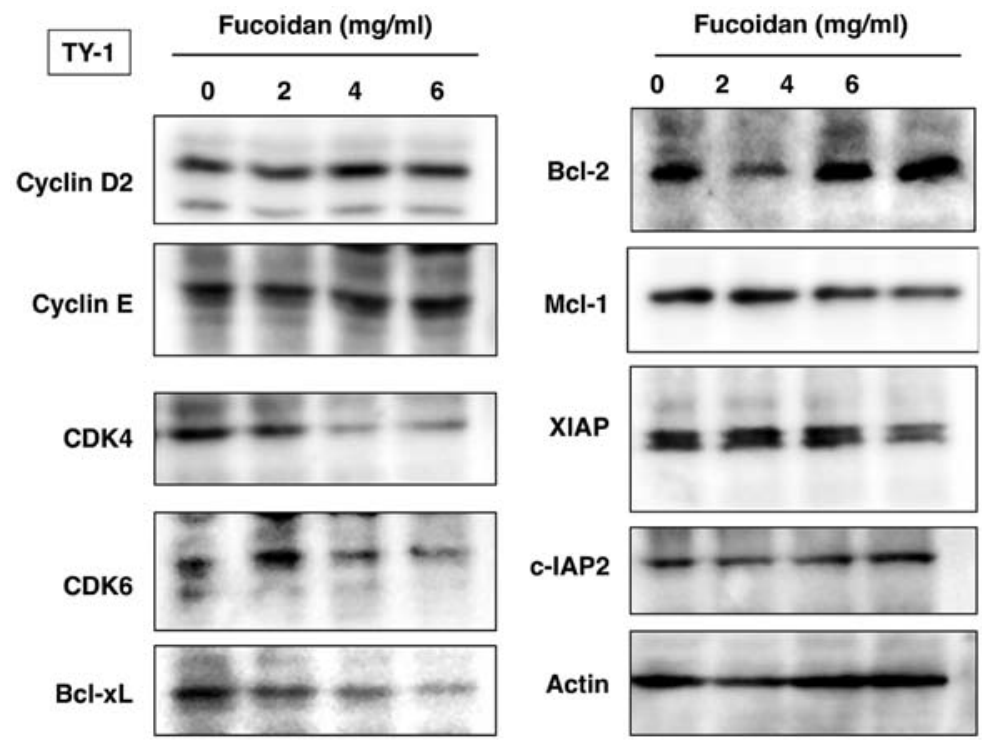

Figure 3. Effects of fucoidan on the expression levels of cellular proteins involved in mitosis and apoptosis. TY-1 cells were treated with different concentrations of fucoidan for $72 \mathrm{~h}$ and the expression levels of the indicated proteins were determined by western blot analysis. Actin was used as an internal control.

Effect of fucoidan on $N F-\kappa B$ activation. In Fig. 4A, we revealed constitutive NF- $\kappa$ B DNA-binding activity in TY-1 and BCBL-1 cells. This binding reaction was specific because NF- $\mathrm{BB}$, but not AP-1 oligonucleotides, competed with the NF- $\kappa B$ DNA-binding activity (Fig. 4A, lanes 2 and 3). The components

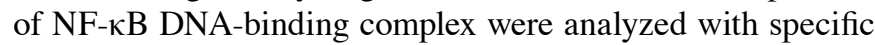

antibodies against five NF- $\mathrm{KB}$ family proteins. Preincubation of nuclear extracts of PEL cells with anti-p50, anti-RelA or anti-RelB antibody caused slow migration of the complex (Fig. 4A, lanes 4, 5 and 8). The results indicate the presence of p50, RelA and RelB in the DNA-binding complex in PEL cells. 
A

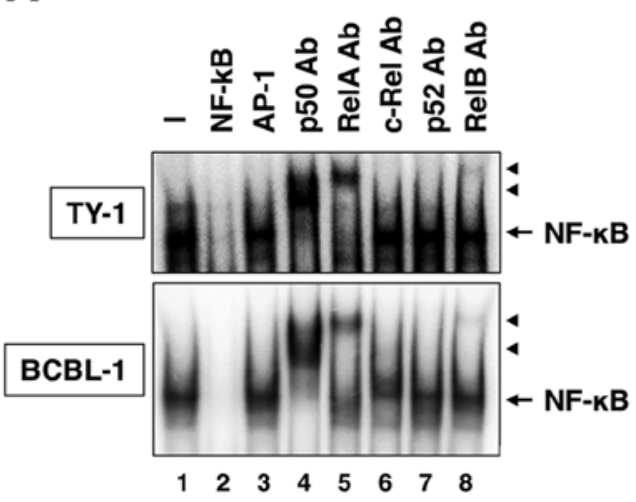

B

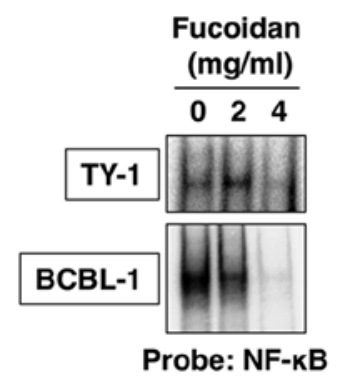

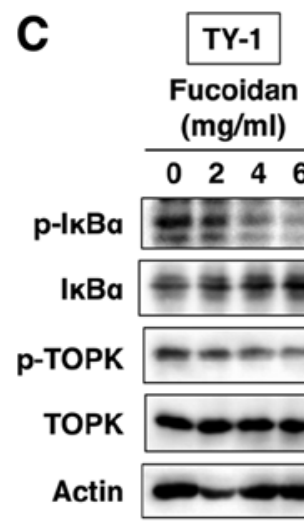

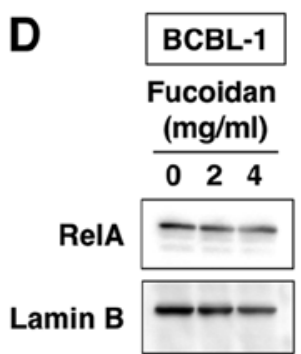

E

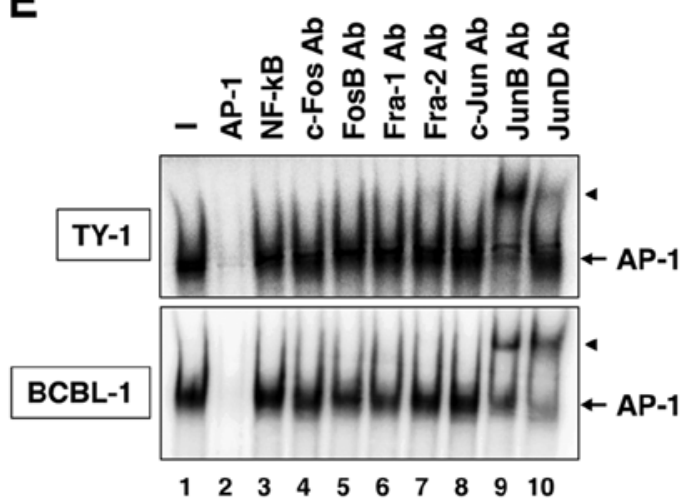

F

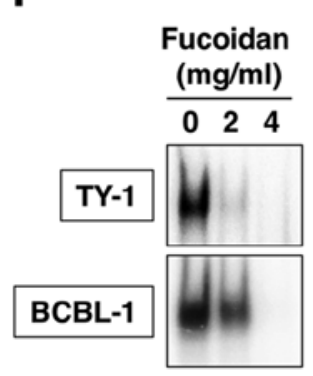

Probe: AP-1
G

TY-1

Fucoidan

$(\mathrm{mg} / \mathrm{ml})$

$\begin{array}{llll}0 & 2 & 4 & 6\end{array}$

JunB $-\ldots$

JunD

Actin

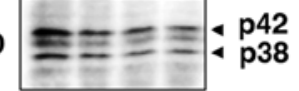

Figure 4. Fucoidan suppresses the NF- $\kappa$ B and AP-1 signaling pathways. (A and E) Constitutive active NF- $\kappa$ B and AP-1 in PEL cells. EMSA was performed to reveal the binding of labeled NF- $\mathrm{BB}$ or AP-1 element to nuclear extracts from TY-1 and BCBL-1 cells (lane 1) and their competition by excess of unlabeled

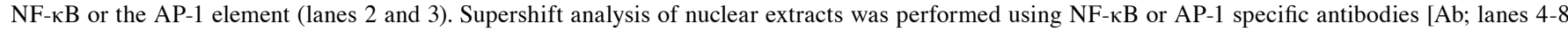
in (A) or lanes 4-10 in (E)]. Arrows indicate the positions of the NF- $\kappa$ B or AP-1 complexes. Arrowheads indicate mobility of the supershifted complexes incubated with antibodies. (B and F) Fucoidan inhibited DNA-binding activities of NF- $\mathrm{B}$ and AP-1. TY-1 and BCBL-1 cells were treated with the indicated concentrations of fucoidan for $48 \mathrm{~h}$. Then, nuclear extracts were prepared, and the DNA-binding activity of NF- $\kappa \mathrm{B}$ or AP-1 was examined using nuclear extracts by EMSA. (C and G) Effects of fucoidan on the NF- $\kappa$ B and AP-1 signaling pathways in PEL cells. TY-1 cells were treated with different concentrations of fucoidan for $48 \mathrm{~h}$. Whole cell lysates were analyzed by western blotting with the indicated antibodies. (D) Inhibition of NF- $\kappa \mathrm{B}$ translocation by fucoidan. BCBL-1 cells were cultured with the indicated concentrations of fucoidan for $48 \mathrm{~h}$. Nuclear fractions were prepared from harvested cells, and RelA nuclear translocation was analyzed by immunoblotting.

To examine the effect of fucoidan on NF-kB DNA-binding activity, we incubated PEL cells with fucoidan $(2$ and $4 \mathrm{mg} / \mathrm{ml})$ for $48 \mathrm{~h}$. The prepared nuclear extracts were analyzed for $\mathrm{NF}-\kappa \mathrm{B}$ DNA-binding activity. The results revealed that fucoidan decreased NF- $\kappa$ B DNA-binding activity in PEL cells (Fig. $4 \mathrm{~B}$ ). $\mathrm{NF}-\kappa \mathrm{B}$ is maintained in an inactive status in the cytoplasm of nonstimulated cells through interaction with specific inhibitor, I $\mathrm{I} \mathrm{B} \alpha(20)$. In response to stimuli, $\mathrm{I} \kappa \mathrm{B} \alpha$ is phosphorylated and degraded through ubiquitin-dependent proteolysis, resulting in the release of free $\mathrm{NF}-\kappa \mathrm{B}$ dimers, which translocate to the nucleus to induce transcription of the target genes (20). That fucoidan inhibited NF- $\kappa$ B DNA-binding activity suggests it may act on NF- $\kappa \mathrm{B}$-associated inhibitory protein I $\mathrm{B} \alpha$. We treated TY-1 cells with fucoidan $(2,4$ and $6 \mathrm{mg} / \mathrm{ml}$ ) for $48 \mathrm{~h}$ and $\mathrm{I} \kappa \mathrm{B} \alpha$ was determined by western blot. Fucoidan dose-dependently inhibited the phosphorylation and degradation of $\mathrm{I} \kappa \mathrm{B} \alpha$ (Fig. 4C). We also examined the suppression of NF- $\kappa \mathrm{B}$ nuclear translocation. The amount of RelA in the nucleus decreased in PEL cells treated with fucoidan for $48 \mathrm{~h}$ (Fig. 4D). Lamin B was used as a quality control to assess nuclear fraction purity and loading levels. Fucoidan did not alter the amount of lamin B in PEL cells (Fig. 4D).
Effect of fucoidan on AP-1 activation. We also investigated the DNA-binding activity of AP-1, another transcription factor, in PEL cells. EMSA demonstrated the constitutive formation of an AP-1 protein complex that retarded the electrophoretic mobility of the AP-1 probe (Fig. 4E). An excess of the cold AP-1 probe abrogated the band, whereas excess of the NF- $\kappa \mathrm{B}$ probe had no effect (Fig. 4E, lanes 2 and 3). Preincubation of nuclear extracts of PEL cells with anti-JunB or anti-JunD antibody caused slow migration of the complex (Fig. 4E, lanes 9 and 10), suggesting that the AP-1 protein complex in PEL cells is composed of JunB and JunD. Fucoidan-treated PEL cells revealed decreased AP-1 DNA-binding activity (Fig. 4F). Furthermore, fucoidan decreased JunB and JunD protein expression in a dose-dependent manner (Fig. 4G).

Effect of fucoidan on TOPK activation. TOPK, a serine-threonine kinase is known to be upregulated in many types of cancer, including lymphoma and leukemia (21). TOPK is a mitotic kinase activated by the CDK1/cyclin B1 complex to promote cytokinesis, and contributes to oncogenic cellular functions (22). Recently, fucoidan was reported to directly interact with TOPK kinase and inhibit its kinase activity (23). 


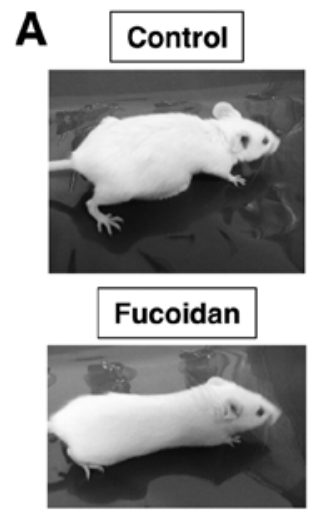

B

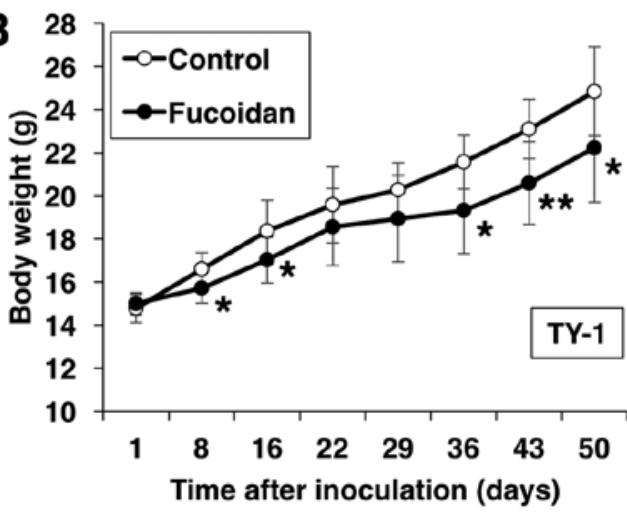

D

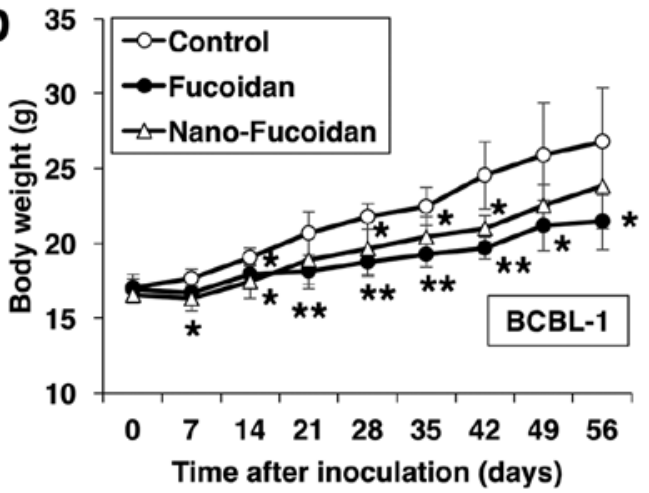

C

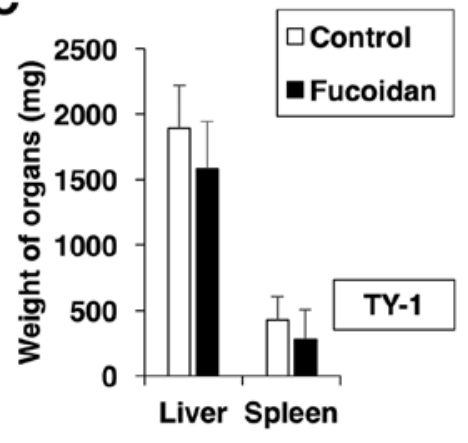

$\mathbf{E}$

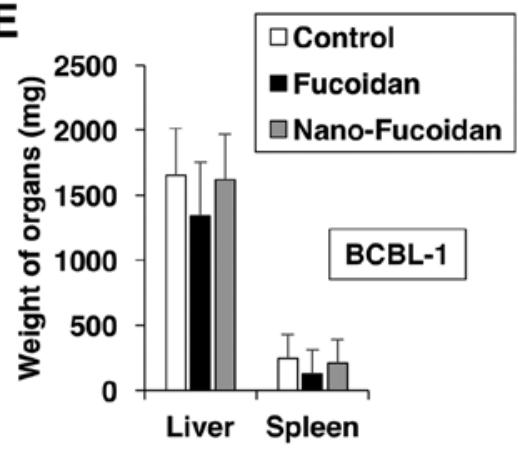

Figure 5. Fucoidan reduces PEL progression in vivo. (A-C) SCID mice were injected intraperitoneally with $5 \times 10^{6}$ TY-1 cells. Beginning the day of injection, $200 \mathrm{mg} / \mathrm{kg}$ fucoidan or vehicle ( $\mathrm{n}=9$ per group) was administered per os, 5 days per week for 50 days. (D and E) Native fucoidan suppressed PEL progression, more potently than nanoparticle fucoidan. SCID mice were injected intraperitoneally with $8 \times 10^{7}$ BCBL-1 cells. Beginning the day after the injection, $150 \mathrm{mg} / \mathrm{kg}$ native or nanoparticle fucoidan, or vehicle ( $\mathrm{n}=5$ per group) were administered per os, 5 days per week for 56 days. (A) Images of representative control and fucoidan-treated mice at the conclusion of the experiments at day 50. (B and D) The body weight was monitored weekly. (C and E) Liver and spleen weights of the control and fucoidan-treated mice. Data are presented as the mean $\pm \mathrm{SD}$. ${ }^{*} \mathrm{P}<0.05$ and ${ }^{* *} \mathrm{P}<0.01$, vs. the vehicle-treated control.

Furthermore, TOPK directly interacted with and phosphorylated IкB $\alpha$ (24). Therefore, we investigated whether fucoidan influenced the activity of TOPK, an upstream kinase of IкB $\alpha$. Fucoidan inhibited the phosphorylation of TOPK but not total TOPK protein expression level (Fig. 4C). TOPK is a mitogen-activated protein kinase (MAPK) kinase-like protein kinase (22). However, fucoidan did not alter the phosphorylation of MAPK, including p38, JNK and ERK (data not shown). These results revealed that TOPK may act as an upstream kinase for I $\mathrm{B} \alpha$ but not MAPK in PEL cells.

Treatment with fucoidan suppresses PEL development in vivo. Finally, we investigated whether fucoidan can suppress PEL development in vivo using an established xenograft murine model. TY-1 cells were injected intraperitoneally into SCID mice followed by oral administration of fucoidan or vehicle for 50 days. Fucoidan-treated mice were slimmer than the control, the latter exhibited massive ascites and expansion of the abdomen (Fig. 5A). Furthermore, the body weight increase of the fucoidan-treated mice throughout the 50-day experiment was less marked than that of the control mice (Fig. 5B). PEL cells express CD30 (2), and sCD30 is produced by PEL cells (25). The ascitic levels of sCD30 revealed a $66 \%$ decrease in fucoidan-treated mice, compared with the control group, albeit statistically insignificant. In addition, fucoidan treatment tended to suppress the increase in liver and spleen weight, albeit insignificantly (Fig. 5C). It has been suggested that different antitumor effects of fucoidan are probably related to the molecular weight of the different preparations $(26,27)$. Therefore, we analyzed the size-dependent bioactivities of fucoidan by comparing the anti-PEL effects of native fucoidan to those of fucoidan lipid nanoparticles in vivo. Relative to the control mice, treatment with native fucoidan and nanoparticle fucoidan resulted in a significant decrease in the rate of body weight increase, although the effect of the former was greater than that of the latter $(\mathrm{P}<0.05$ at 42 days after inoculation; Fig. 5D). The weights of the liver and spleen in native fucoidantreated mice were less than those of either the control or the nanoparticle fucoidan-treated mice (Fig. 5E). The serum levels of sCD30 appear to be a useful biological tumor marker for the diagnosis and management of PEL (25). ELISA used to determine the circulating levels of SCD30 secreted by BCBL-1 cells revealed an 85 and $45 \%$ decrease, respectively, in native fucoidan- and nanofucoidan-treated mice, compared with the control group, albeit statistically insignificant. These results indicate that intraperitoneal inoculation of PEL cells resulted in the development of ascites in SCID mice, and that treatment with fucoidan modulated this process. Furthermore, these effects were dependent on the molecular weight of fucoidan.

\section{Discussion}

Fucoidan, a sulfated polysaccharide, is a constituent of brown algae. It has been extensively studied based on its numerous 
biological activities, including anticoagulation, antiviral, antitumor, immunomodulatory, anti-inflammatory and antioxidant activities $(9,10,28)$. We reported previously the antitumor and antiviral activities of fucoidan extracted from Cladosiphon okamuranus Tokida cultivated in Okinawa (11-14,29). PEL, an aggressive neoplasm caused by KSHV, presents as a lymphomatous effusion in body cavities. To address the potential clinical use of fucoidan, we evaluated the cytotoxic effects of fucoidan on PEL cell lines and determined the molecular mechanism of the anti-PEL effect of fucoidan both in vitro and in vivo.

Our results revealed that fucoidan was cytotoxic against PEL. The cytotoxicity of fucoidan was mediated through cell cycle arrest and apoptosis, as demonstrated by the results of cell cycle analysis, apoptosis and determination of expression levels of cell cycle- and apoptosis-related proteins. Fucoidan inhibited NF- $\mathrm{NB}$ signaling through dephosphorylation of TOPK and $\mathrm{I} \kappa \mathrm{B} \alpha$, and suppressed AP-1 signaling by decreasing JunB and JunD proteins. CDK4, CDK6, Bcl-xL, XIAP and JunB are $\mathrm{NF}-\kappa \mathrm{B}$-regulatory gene products $(30,31)$. Thus, the results revealed that the effects of fucoidan on PEL cells were mediated by dysregulation of various signaling pathways, including NF- $\mathrm{B}$, AP-1, and TOPK signaling. TOPK directly interacted with and phosphorylated I $\kappa \mathrm{B} \alpha$, leading to $\mathrm{NF}-\kappa \mathrm{B}$ activation (24), and NF- $\mathrm{BB}$ elements contributed to JunB inducibility (32). These findings demonstrated that fucoidan targeted TOPK, which led to inactivation of $\mathrm{NF}-\kappa \mathrm{B}$ that is required for AP-1 activation. In addition, the in vivo study revealed that fucoidan exerted anti-PEL activity in SCID mice. In toxicology studies, fucoidan derived from Laminaria japonica and Undaria pinnatifida was found to be safe in rats administered orally at $300 \mathrm{mg} / \mathrm{kg}$ per day for 180 days and at $250-1,000 \mathrm{mg} / \mathrm{kg}$ per day for 28 days, respectively $(33,34)$. In our animal model, fucoidan from Cladosiphon okamuranus Tokida also exhibited little toxicity at $150 \mathrm{mg} / \mathrm{kg}$ per day for 56 days and at $200 \mathrm{mg} / \mathrm{kg}$ per day for 50 days, respectively.

The effects of fucoidan when used in preparations of different molecular weights, remain unknown. We previously demonstrated that the in vivo antitumor activity of fucoidan was significantly higher for nanoparticle fucoidan than for native fucoidan in a xenograft osteosarcoma model (14). However, in the present study, nanoparticle fucoidan had lower anti-PEL activity compared to native fucoidan in the SCID mouse model. High-molecular weight fucoidan has been reported to promote a greater increase in the proportion of cytotoxic $\mathrm{T}$ cells than middle- or low-molecular weight fucoidan in mice which were fed an experimental diet (35). SCID mice have a genetic defect that prevents the functional development of $\mathrm{T}$ and $\mathrm{B}$ lymphocytes, resulting in lack of both $\mathrm{T}$ and $\mathrm{B}$ lymphocytes (36), but have certain residual immunity, such as natural killer (NK) cells, that somehow limits post-transplantation growth and metastasis of human xenografts (37). NK cells play an important role in the control of growth and infiltration of PEL cells (37). It has been reported that oral administration of high-molecular weight fucoidan increased the size of the NK cell population in splenic tissue compared to that observed in either control or intermediatemolecular weight fucoidan (26). Native fucoidan may induce a larger increase in NK cell activity compared with nanoparticle fucoidan in SCID mice. The cell proliferation and survival were decreased in PEL cells treated with relatively higher concentrations of fucoidan in vitro. Fucoidan may exert antiPEL effects indirectly via enhancing immunity in vivo.

Collectively, our results demonstrated that fucoidan has potent anti-PEL activity both in vitro and in vivo. Fucoidan directly inhibited the growth of PEL cells by inducing cell cycle arrest and apoptosis. Future studies should explore the mechanisms of action of fucoidan when used at various molecular weights on PEL cells or immune cells. The results presented here suggest that fucoidan could be potentially useful for the treatment of PEL.

\section{Acknowledgements}

We thank Dr Harutaka Katano for providing TY-1 and BCBL-1, and Kanehide Bio Co. (Okinawa, Japan) for providing native and nanoparticle fucoidan. This study was supported in part by JSPS KAKENHI (grant nos: 15K18414, 25461428 and 17K07175). This study was funded by Kanehide Bio Co. (Okinawa, Japan).

\section{References}

1. Okada S, Goto H and Yotsumoto M: Current status of treatment for primary effusion lymphoma. Intractable Rare Dis Res 3: 65-74, 2014.

2. Nador RG, Cesarman E, Chadburn A, Dawson DB, Ansari MQ, Sald J and Knowles DM: Primary effusion lymphoma: A distinct clinicopathologic entity associated with the Kaposi's sarcomaassociated herpes virus. Blood 88: 645-656, 1996.

3. Chen YB, Rahemtullah A and Hochberg E: Primary effusion lymphoma. Oncologist 12: 569-576, 2007.

4. Carbone A and Gloghini A: KSHV/HHV8-associated lymphomas. Br J Haematol 140: 13-24, 2008.

5. Keller SA, Schattner EJ and Cesarman E: Inhibition of NF-kappaB induces apoptosis of KSHV-infected primary effusion lymphoma cells. Blood 96: 2537-2542, 2000.

6. Gopalakrishnan R, Matta H and Chaudhary PM: A purine scaffold HSP90 inhibitor BIIB021 has selective activity against KSHV-associated primary effusion lymphoma and blocks VFLIP K13-induced NF-кB. Clin Cancer Res 19: 5016-5026, 2013.

7. Cannon ML and Cesarman E: The KSHV G protein-coupled receptor signals via multiple pathways to induce transcription factor activation in primary effusion lymphoma cells. Oncogene 23: 514-523, 2004

8. An J, Sun Y, Sun R and Rettig MB: Kaposi's sarcoma-associated herpesvirus encoded vFLIP induces cellular IL-6 expression: The role of the NF-kappaB and JNK/AP1 pathways. Oncogene 22: 3371-3385, 2003

9. Kwak J-Y: Fucoidan as a marine anticancer agent in preclinical development. Mar Drugs 12: 851-870, 2014.

10. Wang W, Wang S-X and Guan H-S: The antiviral activities and mechanisms of marine polysaccharides: An overview. Mar Drugs 10: 2795-2816, 2012

11. Mori N, Nakasone K, Tomimori K and Ishikawa C: Beneficial effects of fucoidan in patients with chronic hepatitis $C$ virus infection. World J Gastroenterol 18: 2225-2230, 2012.

12. Araya N, Takahashi K, Sato T, Nakamura T, Sawa C, Hasegawa D, Ando H, Aratani S, Yagishita N, Fujii R, et al: Fucoidan therapy decreases the proviral load in patients with human T-lymphotropic virus type-1-associated neurological disease. Antivir Ther 16: 89-98, 2011.

13. Takeda K, Tomimori K, Kimura R, Ishikawa C, Nowling TK and Mori N: Anti-tumor activity of fucoidan is mediated by nitric oxide released from macrophages. Int J Oncol 40: 251-260, 2012.

14. Kimura R, Rokkaku T, Takeda S, Senba M and Mori N: Cytotoxic effects of fucoidan nanoparticles against osteosarcoma. Mar Drugs 11: 4267-4278, 2013.

15. Renne R, Zhong W, Herndier B, McGrath M, Abbey N, Kedes D and Ganem D: Lytic growth of Kaposi's sarcoma-associated herpesvirus (human herpesvirus 8 ) in culture. Nat Med 2: 342-346, 1996. 
16. Katano H, Hoshino Y, Morishita Y, Nakamura T, Satoh H, Iwamoto A, Herndier B and Mori S: Establishing and characterizing a CD30-positive cell line harboring HHV-8 from a primary effusion lymphoma. J Med Virol 58: 394-401, 1999.

17. Mori N and Prager D: Transactivation of the interleukin-1alpha promoter by human T-cell leukemia virus type I and type II Tax proteins. Blood 87: 3410-3417, 1996.

18. Zhang C, Ao Z, Seth A and Schlossman SF: A mitochondrial membrane protein defined by a novel monoclonal antibody is preferentially detected in apoptotic cells. J Immunol 157 : 3980-3987, 1996

19. Khan N, Afaq F and Mukhtar H: Apoptosis by dietary factors: The suicide solution for delaying cancer growth. Carcinogenesis 28: 233-239, 2007.

20. Hayden MS and Ghosh S: Shared principles in NF-kappaB signaling. Cell 132: 344-362, 2008.

21. Simons-Evelyn M, Bailey-Dell K, Toretsky JA, Ross DD, Fenton R, Kalvakolanu D and Rapoport AP: PBK/TOPK is a novel mitotic kinase which is upregulated in Burkitt's lymphoma and other highly proliferative malignant cells. Blood Cells Mol Dis 27: 825-829, 2001.

22. Abe Y, Takeuchi T, Kagawa-Miki L, Ueda N, Shigemoto K, Yasukawa M and Kito K: A mitotic kinase TOPK enhances Cdk1/ cyclin B1-dependent phosphorylation of PRC1 and promotes cytokinesis. J Mol Biol 370: 231-245, 2007.

23. Vishchuk OS, Sun H, Wang Z, Ermakova SP, Xiao J, Lu T, Xue P, Zvyagintseva TN, Xiong H, Shao C, et al: PDZ-binding kinase/T-LAK cell-originated protein kinase is a target of the fucoidan from brown alga Fucus evanescens in the prevention of EGF-induced neoplastic cell transformation and colon cancer growth. Oncotarget 7: 18763-18773, 2016.

24. Park J-H, Yoon D-S, Choi H-J, Hahm D-H and Oh S-M Phosphorylation of I $\mathrm{KB} \alpha$ at serine 32 by T-lymphokine-activated killer cell-originated protein kinase is essential for chemoresistance against doxorubicin in cervical cancer cells. J Biol Chem 288: 3585-3593, 2013

25. Michai M, Goto H, Hattori S, Vaeteewoottacharn K, Wongkham C, Wongkham S and Okada S: Soluble CD30: A possible serum tumor marker for primary effusion lymphoma. Asian Pac J Cancer Prev 13: 4939-4941, 2012.

26. Yang C, Chung D, Shin IS, Lee H, Kim J, Lee Y and You S: Effects of molecular weight and hydrolysis conditions on anticancer activity of fucoidans from sporophyll of Undaria pinnatifida. Int J Biol Macromol 43: 433-437, 2008.
27. Azuma K, Ishihara T, Nakamoto H, Amaha T, Osaki T, Tsuka T, Imagawa T, Minami S, Takashima O, Ifuku S, et al: Effects of oral administration of fucoidan extracted from Cladosiphon okamuranus on tumor growth and survival time in a tumorbearing mouse model. Mar Drugs 10: 2337-2348, 2012.

28. Jiao G, Yu G, Zhang J and Ewart HS: Chemical structures and bioactivities of sulfated polysaccharides from marine algae. Mar Drugs 9: 196-223, 2011.

29. Haneji K, Matsuda T, Tomita M, Kawakami H, Ohshiro K, Uchihara JN, Masuda M, Takasu N, Tanaka Y, Ohta T, et al: Fucoidan extracted from Cladosiphon okamuranus Tokida induces apoptosis of human T-cell leukemia virus type 1-infected T-cell lines and primary adult T-cell leukemia cells. Nutr Cancer 52: 189-201, 2005.

30. Pahl HL: Activators and target genes of Rel/NF-kappaB transcription factors. Oncogene 18: 6853-6866, 1999.

31. Iwanaga R, Ohtani K, Hayashi T and Nakamura M: Molecular mechanism of cell cycle progression induced by the oncogene product Tax of human T-cell leukemia virus type I. Oncogene 20: 2055-2067, 2001

32. Brown RT, Ades IZ and Nordan RP: An acute phase response factor/NF-kappa B site downstream of the junB gene that mediates responsiveness to interleukin- 6 in a murine plasmacytoma. J Biol Chem 270: 31129-31135, 1995.

33. Li N, Zhang Q and Song J: Toxicological evaluation of fucoidan extracted from Laminaria japonica in Wistar rats. Food Chem Toxicol 43: 421-426, 2005.

34. Chung H-J, Jeun J, Houng S-J, Jun H-J, Kweon D-K and Lee S-J: Toxicological evaluation of fucoidan from Undaria pinnatifida in vitro and in vivo. Phytother Res 24: 1078-1083, 2010.

35. Shimizu J, Wada-Funada U, Mano H, Matahira Y, Kawaguchi M and Wada M: Proportion of murine cytotoxic T cells is increased by high molecular-weight fucoidan extracted from Okinawa Mozuku (Cladosiphon okamuranus). J Health Sci 51: 394-397, 2005.

36. Bosma GC, Custer RP and Bosma MJ: A severe combined immunodeficiency mutation in the mouse. Nature 301: 527-530, 1983.

37. Dewan MZ, Terunuma H, Toi M, Tanaka Y, Katano H, Deng X, Abe H, Nakasone T, Mori N, Sata T, et al: Potential role of natural killer cells in controlling growth and infiltration of AIDS-associated primary effusion lymphoma cells. Cancer Sci 97: 1381-1387, 2006. 